\title{
La Cultura Organizacional, Factor Clave para la Transferencia de Conocimiento en los Centros de Investigación del Triángulo del Café de Colombia
}

\author{
Carlos Marulanda ${ }^{(1) *}$, Luis López ${ }^{(1)}$ y Gabriel Cruz $^{(2)}$ \\ (1) Universidad Nacional de Colombia, Facultad de Administración, Departamento de Administración. \\ Campus Palogrande. Calle 27 No. 64-60, Manizales-Colombia. (e-mail: cemarulandae@unal.edu.co; \\ lilopezv@unal.edu.co) \\ (2) Universidad de Caldas, Facultad de Ciencias Agropecuarias, Departamento de Desarrollo Rural y \\ Recursos Naturales. Calle 65 No. 26-10, Manizales-Colombia (e-mail: gabocruz@ucaldas.edu.co). \\ * autor a quien debe ser dirigida la correspondencia
}

Recibido Feb. 20, 2018; Aceptado May. 4, 2018; Versión final May. 22, 2018, Publicado Dic. 2018

\begin{abstract}
Resumen
Se presentan los resultados de un estudio sobre la cultura organizacional como factor clave para la transferencia de conocimiento en los centros e institutos de investigación de los departamentos que forman la región triángulo del café de Colombia. Para el efecto se levantó la información de campo en 24 centros e institutos de investigación. Los hallazgos muestran que hay una valoración alta en cuanto a las patentes, el licenciamiento y el emprendimiento, producto de una cultura organizacional para la transferencia de conocimiento. Se concluye la necesidad de fortalecer la cultura organizacional como factor clave para desarrollar procesos de transferencia de conocimiento de alto nivel. Se recomienda un mayor apoyo de las universidades y de las empresas para aprovechar más lo que se viene haciendo en este campo de la transferencia de conocimiento.
\end{abstract}

Palabras clave: cultura organizacional; transferencia de conocimiento; gestión de conocimiento

\section{The Organizational Culture, a Key Factor for the Transfer of Knowledge in the Coffee Triangle Research Centers of Colombia}

\begin{abstract}
The results of a study about organizational culture as a key factor for the transfer of knowledge in the centers and institutes of investigation of the geographical departments forming the coffee triangle region of Colombia. For this purpose, field information was collected in 24 research centers and institutes. The findings show that there is a high valuation in terms of patents, licensing and entrepreneurship product of an organizational culture for the transfer of knowledge. The need to strengthen the organizational culture as a key factor to develop processes of high-level knowledge transfer is concluded. It is recommended to improve the support of the universities and the companies to take more advantage of what has been done in this field of transfer of knowledge.
\end{abstract}

Keywords: organizational culture; knowledge transfer; knowledge management. 


\section{INTRODUCCIÓN}

El mundo académico y empresarial actual se enfrenta a una serie de retos representados en la alta complejidad, la velocidad de los cambios, el uso de tecnologías de información y comunicaciones, las nuevas formas de producir, la inteligencia artificial, la tercerización, la robotización y la competitividad en aumento de las compañías, buscando la disminución de costos y la mayor rentabilidad. En esta dinámica, las organizaciones que quieren permanecer en el mercado y buscar crecimiento y expansión, se ven obligadas a buscar alternativas que les permitan aprovechar sus recursos y capacidades, es ahí donde aparece la transferencia de conocimiento, como un mecanismo que permite básicamente aprovechar el conocimiento que tienen sus profesionales para convertirlo en capital social y empresarial. Esta transferencia resulta ser más provechosa desde el marco de la innovación y en este sentido, el papel de los centros de investigación es fundamental, para que, en concordancia con los recursos dispuestos por el Gobierno, puedan las empresas alcanzar mayores posibilidades de innovación desde el intercambio y la investigación. Para la exposición de los hallazgos registrados se diseñó y validó un modelo de evaluación de transferencia de conocimiento para centros e institutos de investigación del triángulo del café de Colombia, ubicado en el centro del País, conformado por los departamentos de Caldas, Risaralda y Quindío, presentando seguidamente los resultados referentes a la cultura organizacional para la transferencia de conocimiento de una población de 24 organizaciones.

La cultura organizacional se puede entender como un proceso que implica una combinación de factores como las costumbres, los hábitos, las normas, las reglas, la comunicación formal e informal y todos aquellos que diferencia y hacen única a cada organización. Al respecto Wei y Miraglia (2017), definen cultura organizacional como un concepto multifacético a partir de un patrón de supuestos básicos compartidos aprendidos por un grupo a medida que surgen problemas de adaptación externa y de integración interna, y que ha funcionado lo suficientemente bien como para ser considerado válido y, por lo tanto, para ser enseñado como la manera correcta de percibir, pensar y sentir en relación con los problemas de la organización, el cual se compone de los siguientes elementos principales: artefactos, normas, valores, creencias y supuestos básicos subyacentes. En esta línea, Nazarian et al. (2017), concluyen que la cultura organizacional es el principal recurso que las organizaciones tienen para mantener su ventaja competitiva así mismo representa una combinación de características como el trabajo en equipo, la innovación, la toma de riesgos, la orientación a la capacidad de respuesta del mercado y la satisfacción del cliente, considerando una estructura definida de autoridad con control sobre los flujos de trabajo. En este sentido, Cheung et al., (2011) y Klimas (2016) explican que la cultura transmite un sentido de identidad para los miembros de la organización, facilita la generación de compromiso y aumenta la estabilidad de la organización. De otra parte (Lee et al., 2016; Gallivan y Srite, 2005 y Alaarj et al., 2016) coinciden en que la cultura organizacional se compone de los símbolos, el lenguaje, la ideología, las creencias, los rituales y los mitos de una organización. La cultura es omnipresente y abarca todas las áreas de la vida organizacional; es un núcleo de valores, supuestos, interpretaciones y enfoques que caracterizan a una organización. Y afecta el éxito de las organizaciones influyendo en el comportamiento de los empleados, sus motivaciones y su productividad, así como su propia satisfacción.

Sensuse et al., (2015), plantean igualmente que la cultura organizacional es influenciada por la mentalidad individual y la cultura establecida en cada nivel de la organización y se convierte en un reto para fomentar la eficiencia. El concepto de cultura organizacional se determina como una colección de valores comunes de la organización y las creencias y se manifiesta en la competitividad, la responsabilidad social, el apoyo a la innovación y el rendimiento, y se concibe como una práctica importante para llevar a cabo una implementación exitosa de la transferencia de conocimiento. En la práctica, la alineación de la cultura organizacional con la visión y la misión de la organización conducen a una mejor Implementación del proceso de transferencia de conocimiento. En este sentido Ahmady et al., (2016), establecen que la cultura organizacional influye en la transferencia de conocimiento de cuatro maneras: En el papel que desempeña la cultura organizacional sobre la identidad del conocimiento y su importancia para la organización administración; en el papel que desempeña la cultura para crear una relación entre las personas y el conocimiento de una organización; en los patrones culturales que identifican como el conocimiento tiene que ser usado en una situación especial, y en los procesos que hacen, legitiman y difunden el conocimiento en una organización

Allameh et al., (2011), establecen el concepto de cultura del conocimiento, la cual es una de las ramas específicas de la cultura organizacional; es la indicación de una vía que utiliza a la gente en el proceso de creación e intercambio de información. Además, maneja el conocimiento de otros para lograr los objetivos de la organización y alcanzar el éxito. La cultura afecta los comportamientos que están relacionados con el conocimiento, las personas, los grupos y toda la organización, además tiene una influencia crítica para decidir cuándo, dónde y con quienes debe intercambiarse el conocimiento. La cultura organizacional es considerada como el factor influyente en la gestión del conocimiento y el aprendizaje organizacional; en esta línea, Hernández et al., (2013), explican que un prerrequisito para la evolución del conocimiento tácito es una cultura abierta en una organización que apoye la innovación. La interacción con otros, a diferencia del aislamiento, 
es importante si la conversión del conocimiento tiene lugar. Se encuentran características comunes entre las empresas exitosas de conversión tácita de conocimiento como la resolución de la ambigüedad a través de las comunidades de práctica, la complicidad tácita entre los empleados, las matrices informales de las relaciones entre los empleados y la confianza en el conocimiento colectivo. Complementan lo anterior Durán et al., (2014), y Ahmady et al., (2016), quienes explican que la cultura podría ser un poderoso recurso de propósito común de identidad y directrices flexibles. Su efecto en los miembros de una organización se refleja en el comportamiento, los sentimientos, las percepciones y las actitudes. La transferencia y creación de conocimiento requiere la presencia de la cultura organizacional en la que personas y los grupos tengan que cooperar entre sí y compartan su conocimiento en la dirección de intereses mutuos. En la cultura, el énfasis en el aprendizaje de una organización para crear nuevos conocimientos, ayuda a sus funcionarios a desempeñar un papel activo creando conocimiento al enfatizar el aprendizaje.

Sensuse et al., (2015), concluyen que la cultura organizacional se convierte en un reto para fomentar la limpieza, la burocracia, la eficacia y la eficiencia entre otros, contiene competitividad, responsabilidad social, apoyo a la innovación y al rendimiento; puede ser asumida como un concepto multidimensional y multinivel de valores organizacionales y puede ser creada con el apoyo de la dirección en todos los niveles para el intercambio de conocimientos. El vínculo con la transferencia de conocimiento tiene un gran impacto positivo, de ahí que se convierta en fundamento en la gestión en los diversos niveles de una organización (Zheng et al.,2010; Mueller, 2014 y Trivellas et al., 2015).

Rossia et al., (2017), definen la transferencia de conocimiento como un proceso por el cual el conocimiento se difunde unidireccionalmente de académicos a las partes interesadas externas, que se benefician al usar dicho conocimiento para sus propios objetivos. En este sentido Frishammar et al., (2015), explican que la transferencia de conocimiento se da a través de los límites organizacionales y esta positivamente relacionado con el rendimiento de la empresa, mejora las capacidades innovadoras, aumenta el espacio de innovación y fortalece la ventaja competitiva para asegurar la competencia a largo plazo. Entonces, la transferencia de conocimiento, es el proceso a través del cual también se hace referencia a una transferencia de conocimiento asociada como aprendizaje indirecto de la experiencia de otros, entrando en juego las redes sociales, las tecnologías, las rutinas y la movilidad (Argote y Fahrenkopf, 2016). Según (Chen et al., 2014), el proceso de transferencia de conocimiento requiere compromiso de recursos, tiempo de gestión, atención y esfuerzo y construcción colaborativa. Para hacer que la transferencia de conocimiento sea efectiva, las empresas deben aprovechar su competencia cooperativa, entendida como la capacidad de las organizaciones de interactuar entre empresas entre la empresa focal y con los otros actores empresariales para ajustarse mutuamente a través de la confianza, la comunicación y la coordinación.

(Lorio et al., 2017), evidencian las diferentes formas de actividades de transferencia de conocimiento desde las interacciones entre la universidad y la industria, principalmente en cuanto al intercambio comercial de patentes, licencias y derivaciones. La mayoría de las universidades del mundo han creado instalaciones dedicadas específicamente a la comercialización de invenciones académicas, como parques científicos, oficinas de transferencia de tecnología e incubadoras. Los gobiernos también han apoyado esta forma de interacción universidad-industria al proporcionar fondos para estas instalaciones o subvenciones para proyectos colaborativos. Las contribuciones más recientes han resaltado contratos de investigación y desarrollo, consultoría, intercambio de personal y supervisión conjunta de estudiantes, relaciones de cooperación informales y las actividades de divulgación de conocimientos. En el marco empresarial de acuerdo a lo anterior Liu et al., (2017), explican que la transferencia de conocimiento entre los socios de intercambio es una importante práctica que mejora significativamente la ventaja competitiva de una empresa, a través de la supervisión administrativa, incentivos y programas de capacitación; asimismo, confiar y compartir valores y características de conocimiento, tales como relación, ambigüedad y complejidad.

Los resultados presentados, se apoyan en el modelo de transferencia de conocimiento para centros e institutos de investigación de (Marulanda et al., 2018), el cual se agrupa en 9 categorías: Dirección, cultura, aprendizaje, estándares, tecnologías, redes, servicios, producción comercial y producción intelectual, tal como se puede apreciar en la figura 1; con sus 25 variables: direccionamiento, estructura, reglas de propiedad intelectual, valores, clima, orientación, estrategia, competencias, roles, métodos, tecnologías de información - TI, organizaciones, investigación, contratos, alianzas, asesorías, consultorías, capacitaciones, patenteslicenciamiento, emprendimiento, artículos, libros, tesis, programas y ponencias.

\section{Metodología}

La metodología se basó en el estudio de (Marulanda et., 2018), con base en los siguientes elementos:

Delimitación de las unidades de observación: Básicamente las unidades de observación fueron los centros e institutos de investigación de la región triángulo del café de Colombia, de los cuales se logró integrar una 
población analizada de 24 .

Escala de medida: Se formularon preguntas basadas en la escala de Likert, las cuales fueron calificadas de 1 a 5 , donde la categorización es la siguiente: 1, se está en desacuerdo o no realizado, 2, realizado parcialmente, 3 , realizado en intervalos de tiempo, 4 , realizado con regularidad y 5 realizado completamente. El grado de realización se refirió al impacto calificado de la variable respecto a la transferencia de conocimiento en las organizaciones.

Categorías y variables evaluadas: De acuerdo a las características de valoración, se agruparon las preguntas en las siguientes categorías y variables respecto a la cultura organizacional. Ver tabla 1.

Tabla1: Categorías y variables

\begin{tabular}{|l|l|}
\hline \multicolumn{1}{|c|}{ Categorías } & \multicolumn{1}{c|}{ Variables } \\
\hline Cultura & Valores y clima organizacional \\
\hline Servicios & Asesoría, consultoría y capacitación \\
\hline Producción comercial & Patentes-licenciamiento y emprendimiento \\
\hline
\end{tabular}

Basados en los planteamientos de Hair et al., (2007), frente a la evaluación de constructos teóricos, se considera el uso del análisis de correlación, el cual implica relacionar simultáneamente las variables establecidas entre sí, y resumir los resultados en una matriz de correlaciones.

\section{RESULTADOS Y DISCUSIÓN}

Los resultados que se presentan a continuación se relacionan con la categoría cultura organizacional, en los centros e institutos de investigación del triángulo del café de Colombia, que consideran las siguientes variables: Valores (valor) y clima organizacional (clima). Los resultados se enseñan en la figura 1.

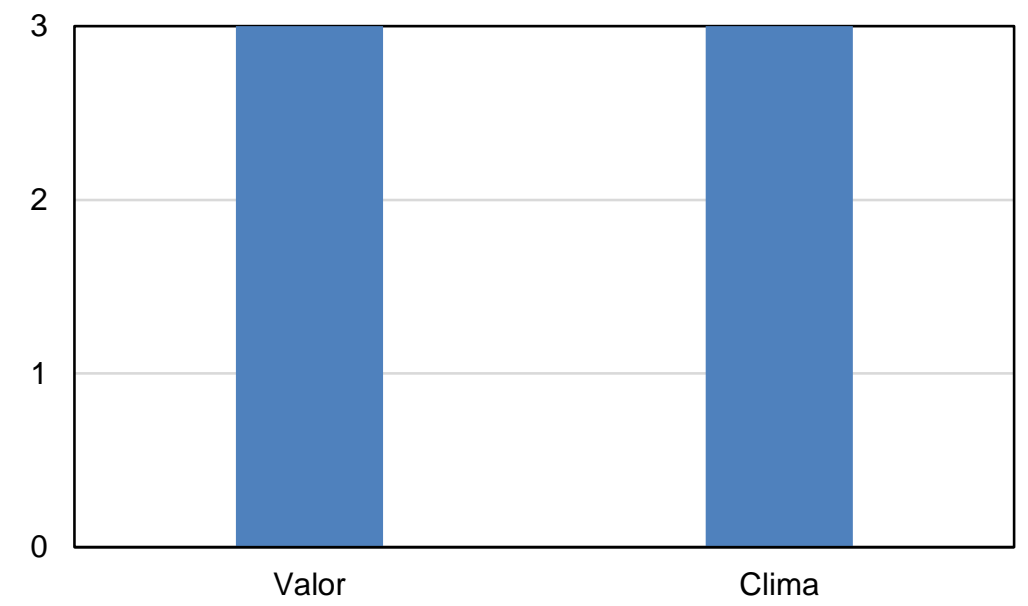

Fig. 1: Valoración de las variables de la categoría cultura organizacional

Los resultados registrados, muestran una valoración homogénea de 3,0, sobre 5,0, tanto para los valores como para el clima organizacional, lo que refiere una calificación media de las variables analizadas respecto a la transferencia de conocimiento en la cultura organizacional. El resultado se interpreta como de calificación positiva, y que pese a todas las circunstancias que se hayan podido presentar en los centros e institutos de investigación, las personas que hacen parte de éstos, están haciendo las cosas en la dirección del camino de la transferencia, es decir, están aportando desde el quehacer diario, y desde los procesos y procedimientos cumplidos, a desarrollar una transferencia de conocimiento importante con sus pares empresariales.

En cuanto a la categoría de producción comercial y servicios en los centros e institutos de investigación del triángulo del café de Colombia, se consideraron las siguientes variables de medición: Asesoría (asesor), Consultoría (consult), capacitación (capacit), patentes-licenciamiento (patent) y emprendimiento (emprend). Los resultados se observan en la figura 2. 


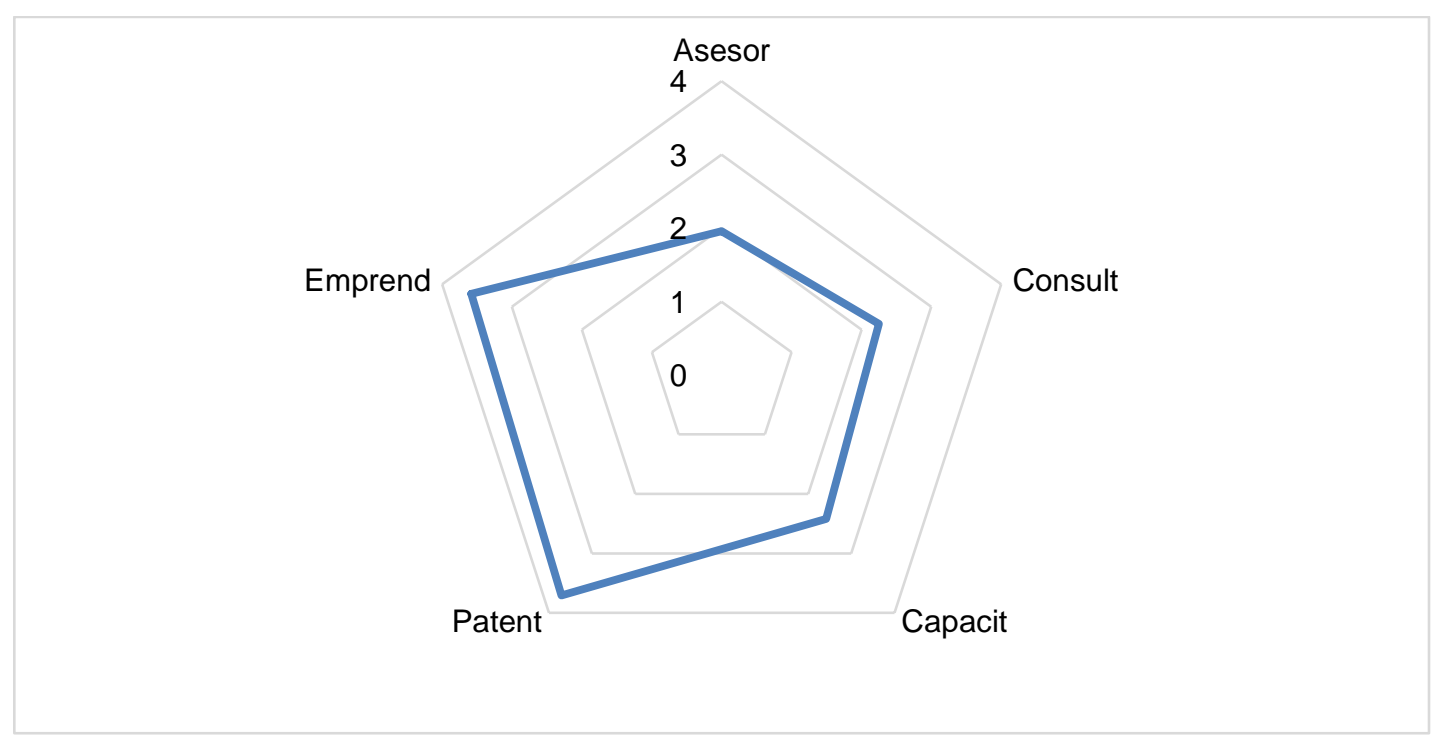

Fig. 2: Valoración de las variables de la categoría servicios

Los hallazgos encontrados muestran una valoración así: aproximándose a 4,0 las patentes, el licenciamiento y los emprendimientos resultados de los trabajos de investigación en los centros e institutos de investigación, hallazgos muy importantes dada la naturaleza y función de tales organizaciones y las respuestas que deben entregar a las empresas, lo que indudablemente se revierte en una mayor riqueza social y en un mayor desarrollo y apoyo a la investigación desde estos centros. Ahora bien, aproximándose a 2 en la valoración se encuentran las asesorías, consultorías y capacitaciones, lo que muestra poco desarrollo e influencia de éstos factores, observando la necesidad de explorarlos más y ofrecer mayores posibilidades en relación con las necesidades de las organizaciones vinculadas.

Considerando los anteriores resultados se realizó un análisis de correlación, para determinar la asociación o relación entre las variables evaluadas, cuyos coeficientes de correlación se observan en la matriz de correlaciones enseñada en la tabla 2. En la Tabla, (*) indica que la correlación es significativa en el nivel 0,05 (prueba a 2 colas; $\left({ }^{*}\right)$ in dica que la correlación es significativa en el nivel 0,01 (prueba a 2 colas; $y$ sin * significa que nNo se prueba la correlación. Los resultados de la prueba de correlación muestran que existe una relación directa y significativa entre las categorías y las variables valoradas, ya que los resultados expresan valores significativos por encima del $30 \%$.

Tabla 2: Matriz de correlación de variables.

\begin{tabular}{|l|c|c|c|c|c|c|c|}
\hline Correlaciones & Valores & Clima & Patentes-lic & Emprendimiento & Asesorías & Consultorías & Capacitaciones \\
\hline Valores & 1 & & & & & & \\
\hline Clima &, $451^{*}$ & 1 & & & & & \\
\hline Patentes-lic &,- 217 &, 279 & 1 & & & & \\
\hline Emprendimiento &,- 121 &, 325 &, $631^{* *}$ & 1 & & & \\
\hline Asesorías &,- 035 &, $620^{* *}$ &, 215 &, 288 & 1 & & \\
\hline Consultorías &, 000 &, $472^{*}$ &, 035 &, 132 &, $764^{* *}$ & 1 & \\
\hline Capacitaciones &,- 025 &, $580^{* *}$ &, 240 &, $426^{*}$ &, $842^{* *}$ &, $743^{* *}$ & 1 \\
\hline
\end{tabular}

Los anteriores resultados van en la misma línea de (Murad y Park, 2016), quienes reconocen la importancia de las competencias no técnicas y organizacionales y factores tales como: habilidades de gestión, capacidades organizacionales, cultura, procesos y rutinas e información, en procesos de transferencia de conocimiento. Los mismos también siguen lo planteado por Vick y Popadiuk (2015), quienes concluyen que los equipos caracterizados por una cultura de conocimiento asumen un enfoque en la información externa, derivado del contacto frecuente con la empresa asociada. En estos casos, los valores de información promueven la audacia de los individuos para tomar la iniciativa; y por lo tanto se admite que este tipo de cultura de la información promueve la externalización del conocimiento, ya que se anima a los individuos a explorar nuevas ideas y conocimiento exógeno, del tácito al explícito para el equipo. 
Se coincide además con lo encontrado por (Wang et al, 2014), quienes establecen que el dominio de la colaboración está dado con la participación de la información, la planificación conjunta, la resolución de problemas, la medición conjunta del desempeño y el aprovechamiento de recursos y habilidades, en el marco de la transferencia de conocimiento. También con lo planteado por Lee et al., (2016), quienes concluyeron que la cultura organizacional afecta decisivamente el intercambio de conocimientos, y consideran a la cultura organizacional como un factor en la implementación de la transferencia de conocimiento. Asimismo, Chumg et al., (2016), explican que la cultura organizacional es la conciencia compartida de los colegas con supuestos compartidos, referidos a que pueden influir en el comportamiento, pensamientos y percepciones de los miembros), valores, creencias y normas, además de las interpretaciones suscitadas.

La cultura es entonces considerada como un factor crítico que influye fuertemente en la conducta de transferencia de conocimientos en las organizaciones. Así mismo (García-Barriocanal et al., 2012) establecen que el conocimiento es un recurso de la base de las capacidades de la empresa, es decir, se convierte en competencias empresariales; tales competencias son categorizadas como conocimiento relacionado con el trabajo. Finalmente se coincide con Hussain et al., (2016), quienes concluyen que conocer las necesidades de los clientes, hace hincapié en la transferencia de conocimientos como la relación equipo-cultura, para influir en el rendimiento y en la innovación de los servicios ofertados.

Estos resultados alineados con otros resultados de investigación relacionados con la cultura organizacional y la transferencia de conocimiento, los cuales se explican en la tabla 3:

Tabla 3: Otros resultados de investigación

\begin{tabular}{|c|c|}
\hline Autor & Conclusiones \\
\hline Marulanda et al., (2016) & $\begin{array}{l}\text { Existe una relación directa asociada a la apropiación de la gestión de conocimiento } \\
\text { relacionada con una cultura organizacional. Es necesario apropiar y transferir } \\
\text { conocimiento por parte de los individuos, en el marco de una cultura organizacional } \\
\text { que propicie estrategias y propuestas de valor. }\end{array}$ \\
\hline (Franco y Pinho 2017) & $\begin{array}{l}\text { La transferencia de conocimiento brinda a los centros de investigación un acceso } \\
\text { más rápido a conocimiento poseído por investigadores y así se encuentran } \\
\text { soluciones a problemas comunes. Lo que permite satisfacer las necesidades del } \\
\text { mercado, a través de la creación de nuevos productos o tecnología desarrollada } \\
\text { por los investigadores. Desde la cooperación como factor definitivo en el marco de } \\
\text { la cultura organizacional. }\end{array}$ \\
\hline (Vlajčić et al., 2018) & $\begin{array}{l}\text { La cultura organizacional tiene un efecto positivo en la transferencia de } \\
\text { conocimiento. Desde la creación, codificación y decodificación del conocimiento. }\end{array}$ \\
\hline (Ado et al., 2017) & $\begin{array}{l}\text { La gestión de la cultura juega un papel clave en el aprendizaje, la transferencia de } \\
\text { conocimiento y desarrollo del capital social. Es importante el manejo oportuno de } \\
\text { las diferencias culturales en el desarrollo de estrategias y tácticas para obtener } \\
\text { conocimiento y generar aprendizaje. }\end{array}$ \\
\hline (Chih-Hsing, 2018) & $\begin{array}{l}\text { En el marco de la cultura organizacional el aprendizaje es un desafío crítico desde } \\
\text { los procesos de capital social y requiere de un análisis importante para que los } \\
\text { gerentes de PYME entiendan mejor este fenómeno y lograr una estrategia efectiva } \\
\text { para transferencia de conocimiento. }\end{array}$ \\
\hline (Wei y Miraglia, 2017) & $\begin{array}{l}\text { La evidencia emergente contribuye a profundizar nuestra comprensión de la } \\
\text { dinámica sutil a través de la cual elementos culturales que operan en varios niveles } \\
\text { organizacionales contribuyen a orientar, legitimar, apoyar o desalentar decisiones, } \\
\text { acciones e interacciones de los individuos en términos de intercambio de } \\
\text { conocimiento, transferencia y reutilización. El impacto de la cultura organizacional } \\
\text { en la transferencia de conocimiento a múltiples niveles organizacionales es } \\
\text { relativamente poderoso. }\end{array}$ \\
\hline
\end{tabular}

\section{CONCLUSIONES}

Del correspondiente análisis y discusión de los resultados, se desprenden las siguientes conclusiones: (i) Existen algunos avances importantes en el desarrollo de una cultura organizacional para la transferencia de conocimiento en las unidades de análisis, pero es necesario priorizarla y robustecerla; (ii) se obtienen buenos resultados con respecto a las patentes, el licenciamiento y los emprendimientos resultados de la investigación, pero falta por hacer sobre el proceso de ofrecer más y mejores servicios de capacitación, asesorías y 
consultorías. iii) Los resultados de las pruebas de correlación registran deficiencias en cuanto a los servicios de asesorías, consultorías y capacitación, lo cual es parcialmente explicable por factores como la falta de personal preparado y/o de recursos especializados para este fin, y (iv) Se requiere un enfoque diferente respecto al tema de la investigación y el portafolio de servicios que ofrecen los centros e institutos de investigación del triángulo del café de Colombia.

\section{AGRADECIMIENTOS}

A la Dirección de Investigaciones de la Universidad Nacional sede Manizales DIMA. Nombre de la convocatoria: Convocatoria nacional de proyectos para el fortalecimiento de la investigación, creación e innovación de la Universidad Nacional de Colombia. Proyecto de Investigación titulado: La transferencia de conocimiento en centros e institutos de investigación del triángulo del café. Código Hermes: 35721.

\section{REFERENCIAS}

Ado, A., Z. Su y R. Wanjiru, Learning and Knowledge Transfer in Africa-China JVs: Interplay between Informalities, Culture, and Social Capital, Journal of International Management, (23), 166-179 (2017)

Ahmady, G., A. Nikooravesh y M. Mehrpour, Effect of organizational culture on knowledge management based on Denison model, Procedia - Social and Behavioral Sciences, (230), 387 - 395 (2016)

Alaarj, S., Z. Abidin y U. Ahmad, Mediating Role of Trust on the Effects of Knowledge Management Capabilities on Organizational Performance, (I. 2.-3. 12th International Strategic Management Conference, Ed.) Procedia - Social and Behavioral Sciences, (235), 729 - 738 (2016)

Allameh, M., M. Zamanib y S. Reza, The Relationship between Organizational Culture and Knowledge Management, Procedia Computer Science, (3), 1224-1236 (2011)

Argote, L. y E. Fahrenkopf, Knowledge transfer in organizations: The roles of members, tasks, tools, and networks, Organizational Behavior and Human Decision Processes, (136) 146-159 (2016)

Chen, C.-J., Y.-C. Hsiao y M.-A. Chu, Transfer mechanisms and knowledge transfer: The cooperative competency perspective, Journal of Business Research, (67), 2531-2541 (2014)

Cheung, S., P. Wong y W. Ada, Towards an organizational culture framework in construction, International Journal of Project Management, (29), 33-44 (2011)

Chih-Hsing, S., Examining social capital, organizational learning and knowledge transfer in cultural and creative industries of practice, Tourism Management, 64, 258-270 (2018)

Chumg, H.-F., J. Seaton, L. Cooke y W.-Y. Ding, Factors affecting employees' knowledge-sharing behaviour in the virtual organisation from the perspectives of well-being and organisational behavior, Computers in Human Behavior, (64), 432$448(2016)$

Duran, C., A. Çetindere y Ö. Şahan, An analysis on the relationship between total quality management practices and knowledge management: The case of Eskişehir, Procedia - Social and Behavioral Sciences, (109), 65 - 77 (2014)

Franco, M. y C. A. Pinho, Case study about cooperation between University Research Centres: Knowledge transfer perspective, Journal of Innovation \& Knowledge, 9-18 (2017)

Frishammar, J., K. Ericsson y P. Patel, The dark side of knowledge transfer: Exploring knowledge leakage in joint R\&D projects, Technovation, 41(42), 75-88 (2015)

Gallivan, M. y M. Srite, Information technology and culture: Identifying fragmentary and holistic perspectives of culture, Information and Organization, (15), 295-338 (2005)

García-Barriocanal, E., M.-A. Sicilia y S. Sánchez-Alonso, Computing with competencies: Modelling organizational capacities, Expert Systems with Applications, (39), 12310-12318 (2012)

Hair, J., R. Anderson, R. Tatham y W. Black, Análisis multivariante, Madrid, Pearson (2007)

Hernández, J., Y. Hernández, D. Collado y D. Cebrián, Knowledge Creating and Sharing Corporate Culture Framework, Procedia - Social and Behavioral Sciences, 388 - 397 (2013)

Hussain, K., R. Konar y F. Ali, Measuring Service Innovation Performance through Team Culture and Knowledge Sharing Behaviour in Hotel Services: A PLS Approach, Procedia - Social and Behavioral Sciences, (224), 35 - 43 (2016)

Klimas, P., Organizational culture and coopetition: An exploratory study of the features, models and role in the Polish Aviation Industry, Industrial Marketing Management, (53), 91-102 (2016)

Lee, J.-C., Y.-C. Shiue y C.-Y. Chen, Examining the impacts of organizational culture and top management support of knowledge sharing on the success of software process improvement, Computers in Human Behavior, (54), 462-474 (2016)

Liu, Y., Y. Li, L. Hui y T. Liu, Knowledge transfer in buyer-supplier relationships: The role of transactional and relational governance mechanisms, Journal of Business Research, (78), 285-293 (2017) 
Lorio, R., S. Labory y F. Rentocchini, The importance of pro-social behaviour for the breadth and depth of knowledge transfer activities: An analysis of Italian academic scientists, Research Policy, (46), 497-509 (2017)

Marulanda, C., O. Bedoya y H. Quintero, Modelo de transferencia de conocimiento para centros e institutos de investigación, Revista Espacios, (39), 1-20 (2018)

Marulanda, C., M. López y F. López, La Cultura Organizacional y las Competencias para la Gestión del Conocimiento en las Pequeñas y Medianas Empresas (PYMEs) de Colombia, Inf. Tecnol., 27 (6) 03-10 (2016)

Mueller, J., A specific knowledge culture: Cultural antecedents for knowledge sharing between project teams, European Management Journal, (32), 190-202 (2014)

Murad, A., y K. Park, The mediating role of an innovative culture in the relationship between absorptive capacity and technical and non-technical innovation, Journal of Business Research, (69), 1669-1675 (2016)

Nazarian, A., P. Atkinson y P. Foroudi, Influence of national culture and balanced organizational culture on the hotel industry's performance, International Journal of Hospitality Management, (63), 22-32 (2017)

Rossia, F., A. Rosli y N. Yip, Academic engagement as knowledge co-production and implications for impact: Evidence from Knowledge Transfer Partnerships, Journal of Business Research, (80), 1-9 (2017)

Sensuse, D., E. Cahyaningsih y W. Wibowo, Knowledge Management: Organizational Culture in Indonesian Government Human Capital Management. Procedia Computer Science, (72), 485 - 494 (2015)

Trivellas, P., Z. Akrivouli, E. Tsifora y P. Tsoutsa, The impact of knowledge sharing culture on job satisfaction in accounting firms, The mediating effect of general competencies, Procedia Economics and Finance, (19), 238 - 247 (2015)

Vlajčić, D., A. Caputo, G. Marzi y M. Dabić, Expatriates managers' cultural intelligence as promoter of knowledge transfer in multinational companies, Journal of Business Research, 1-11 (2018)

Vick, T. E. y S. Popadiuk, Information culture and its influences in knowledge creation: Evidence from university teams engaged in collaborative innovation projects, International Journal of Information Management, 292-298 (2015)

Wang, E., F. Chou, N. Lee y S. Lai, Can intrafirm IT skills benefit interfirm integration and performance? Information \& Management, (51), 924-938 (2014)

Wei, Y. y S. Miraglia, Organizational culture and knowledge transfer in project-based organizations: Theoretical insights from a Chinese construction firm, International Journal of Project Management, (35), 571-585 (2017)

Zheng, W., B. Yang y G. McLean, Linking organizational culture, structure, strategy, and organizational effectiveness: Mediating role of knowledge management, Journal of Business Research, (63), 763-771 (2010) 